\title{
Ergodicity breaking and quasistationary states in systems with long-range interactions
}

\author{
Ana C. Ribeiro-Teixeira, Fernanda P. C. Benetti, Renato Pakter, and Yan Levin \\ Instituto de Física, Universidade Federal do Rio Grande do Sul Caixa Postal 15051, CEP 91501-970, Porto Alegre, RS, Brazil
}

(Received 30 October 2013; published 21 February 2014)

\begin{abstract}
In the thermodynamic limit, systems with long-range interactions do not relax to equilibrium, but become trapped in quasistationary states (qSS), the life time of which diverges with the number of particles. In this paper we will explore the relaxation of the Hamiltonian Mean-Field model to qSS for a class of initial conditions of the multilevel water-bag form. We will show that if the initial distribution satisfies the virial condition, thereby reducing mean field changes, the final distribution in the qSS can be predicted very accurately using a reduced exactly integrable model. The calculated distribution functions obtained using this approach are found to be more accurate than the ones predicted by the Lynden-Bell theory.
\end{abstract}

DOI: 10.1103/PhysRevE.89.022130

PACS number(s): 05.20.-y, 05.45.-a, 05.70.Ln

\section{INTRODUCTION}

Long-range interacting (LRI) systems are ubiquitous in nature. They range from the astronomical scale [1-3], to the macroscopic, e.g., non-neutral plasmas [4,5], wave-plasma interacting systems (free-electron lasers) [6,7], and twodimensional geophysical vortex systems [8], down to the atomic scale, e.g., classical and quantum cold atoms interacting via quasiresonant lasers $[9,10]$. In spite of their importance, much of the behavior of these systems remains poorly understood (for recent reviews, see, e.g., Refs. [11-13]). It is known that LRI systems can exhibit ergodicity breaking [14-17], anomalous relaxation and diffusion [18,19], quasistationary states (qSS) [20,21], vanishing Lyapunov exponents, inequivalence of ensembles [22-24], negative specific heat (nonconcave microcanonical entropies) [25,26], temperature discontinuities, etc. The thermodynamic anomalies result from the nonadditivity of energy, while the dynamical pathologies arise from the complexity of collisionless relaxation driven by the wave-particle interactions.

In the thermodynamic limit $N \rightarrow \infty$, the dynamics of systems with long-range forces is governed by the collisionless Boltzmann, or Vlasov, equation:

$$
\begin{gathered}
\frac{\partial f}{\partial t}+\mathbf{p} \cdot \nabla_{\mathbf{q}} f-\nabla_{\mathbf{q}} V[f](\mathbf{q}, t) \cdot \nabla_{\mathbf{p}} f=0, \\
V[f](\mathbf{q}, t) \equiv \iint d \mathbf{q}^{\prime} d \mathbf{p}^{\prime} f\left(\mathbf{q}^{\prime}, \mathbf{p}^{\prime}, t\right) v\left(\left|\mathbf{q}-\mathbf{q}^{\prime}\right|\right),
\end{gathered}
$$

where $f(\mathbf{q}, \mathbf{p}, t)$ is the one-particle distribution function and where $v\left(\left|\mathbf{q}-\mathbf{q}^{\prime}\right|\right)$ is the two-body microscopic interaction potential. Note that the mean-field potential $V[f](q, t)$ is a functional of the one-particle distribution $f(\mathbf{q}, \mathbf{p}, t)$. This makes the Vlasov equation (1) nonlinear and very difficult to solve. Equation (2) shows that the mean-field potential and the distribution function dynamics are intertwined.

The dynamics of a large, but finite, $N$ system may also be well approximated by the Vlasov equation up to time $t_{\max }$. Indeed, Braun and Hepp's theorem [27] states that for a twobody smooth long-range potential the "distance" (in the space of all measures) between two initially close solutions of the Vlasov equation increases at most exponentially in time, which establishes a lower bound $t_{\max }=O[\ln (N)]$ for the time up to which the molecular dynamics (MD) evolution of a finite $N$
LRI system will be described by its associated Vlasov flow. ${ }^{1}$ In fact, qSS (corresponding to stable stationary states of the Vlasov equation) persist for times that grow as a power of $N$ [21]. Therefore, for LRI systems the $\lim N \rightarrow \infty$ and $\lim t \rightarrow$ $\infty$ do not commute; taking the latter limit before the former, the system should reach thermodynamic equilibrium, while taking the former before the latter, Vlasov dynamics is always valid and thermodynamic equilibrium is not reached.

For LRI systems with a finite number of particles, the strongly oscillating mean-field potential and the resulting parametric resonances lead to a fast relaxation to qSS, on a time scale independent of $N$. This is known in astrophysics as a violent relaxation [28]. The time reversibility of the Vlasov equation implies that the phase space evolution continues indefinitely on progressively smaller scales. Therefore, no invariant fine-grained measure can ever be reached. Filamentation of the distribution function occurs because initially neighboring phase space elements will evolve according to distinct phase velocities. Evolution deforms initial condition support through ever finer filamentations. The coarse-grained distribution function $\bar{f}$, on the other hand, approaches a stationarity. The concept of qSS and of entropy production are valid only on a coarse-grained level $\bar{f}(\mathbf{q}, \mathbf{p}, t)$. In the limit $N \rightarrow \infty$, the mean-field potential in the qSS will become stationary, while for finite $N$ small oscillations will persist indefinitely. These fluctuations will eventually drive a finite system out of the qSS and to the Boltzmann-Gibbs equilibrium. In this paper we will not be interested in the finite $N$ corrections to the Vlasov dynamics, restricting ourselves to the time scale shorter than the lifetime of the qSS.

The determination of stable solutions to the Vlasov dynamics is not an easy task. Besides the usual constants of motion such as energy, linear and angular momentum, Vlasov

\footnotetext{
${ }^{1}$ The initial error of a large $N$ particle approximation $f_{N}(\mathbf{q}, \mathbf{p}, t)$ of a continuous distribution $f(\mathbf{q}, \mathbf{p}, t)$ is $d_{0}=O(1 / \sqrt{N})$, on account of the central limit theorem. Hence, since Braun-Hepp's theorem is also valid for weak solutions, the maximum time during which the molecular dynamics discrete distribution may, within an error $\epsilon$, coincide with its associated Vlasov description grows as $\ln (N)$ at least,
}

$$
d\left(f_{N}, f\right) \lesssim d_{0} e^{\alpha t} \leqslant \epsilon \rightarrow t_{\max } \geqslant O[\ln (N)]
$$


dynamics has an infinite number of conserved quantities known as the Casimir invariants or simply the Casimirs $[29,30]$,

$$
C_{s}[f]=\int s[f(\mathbf{q}, \mathbf{p}, t)] d \mathbf{q} d \mathbf{p},
$$

where $s(x)$ is an arbitrary functional. Their conservation is equivalent to the conservation of phase space densities, which are a special case of Casimirs. The Casimirs (3) represent an infinity of conserved quantities. A given initial condition will select from the start of the evolution a given invariant submanifold, which will correspond to one of the stable stationary states of the dynamics. Even if we were able to know all solutions (invariant submanifolds) to the Vlasov equation, we would still need to determine to which invariant submanifold a given initial condition would correspond.

The nonlinearity and the infinity of integrals of motion complicate the study of LRI systems. To circumvent the necessity of integrating the Vlasov equation to obtain the final stationary state, Lynden-Bell (LB) proposed a Boltzmann-like statistical approach. LB argued that the qSS should correspond to the maximum of the coarse-grained entropy [28]. For this to work, however, requires that the dynamics of a LRI system must be ergodic and mixing, which in general is not the case. Although simple and elegant, the LB theory in general is not able to predict accurately the particle distribution inside the qSS [13,31-38]. It was observed, however, that if the initial distribution satisfies the virial condition, and the oscillations of the mean-field potential are suppressed, the qSS marginal distribution functions predicted by the LB theory are in excellent agreement with the results of molecular dynamics simulations $[2,3,5,17,35,39]$. The virial theorem establishes a stable stationarity condition for a system of bound interacting particles. For an isolated system starting from an arbitrary initial condition, its kinetic and potential energies will oscillate around the corresponding virial condition, progressively approaching it as the system approaches stationarity. The closer the system is to such virial condition, the smaller is the amplitude of the mean-field potential oscillations.

On the other hand, if the initial distribution does not satisfy the virial condition, the mean-field potential undergoes violent oscillations. Some particles can then enter in resonance with the macroscopic oscillations gaining large amounts of energy, thus populating the regions of the phase space that are highly improbable from the perspective of LB or Maxwell-Boltzmann statistics, forming a thin halo. The particle evaporation produced by the resonances takes away energy from the collective motion leading to Landau damping [40-42] of the macroscopic oscillations of the mean-field potential. If the oscillations die completely, the mean-field potential becomes static and the dynamics of each particle becomes integrable (for systems with one degree of freedom). The final qSS reached by LRI systems is not ergodic, with the particle distribution often characterized by a "cold" dense core and a "hot" tenuous halo of evaporated (resonant) particles [13]. On the other hand, a good agreement between the LB theory and MD simulations for initial distributions satisfying the virial condition has been attributed to the existence of ergodicity and mixing. This, however, is paradoxical. As has been discussed above, the relaxation dynamics of LRI systems is driven by the fluctuations of the mean-field potential. If, on the other hand, the initial distribution function satisfies the virial condition, the oscillations will be suppressed diminishing the mixing of different phase space levels, which should lead to poor mixing and lack of ergodicity.

Recently, an approach very different in spirit to LB theory has been proposed to account for the qSS attained by the Hamiltonian Mean-Field (HMF) model [43-45]. Since the qSS are characterized by the virialization of the distribution, i.e., by the stationarity of the mean-field potential and consequent integrability of the model, it might be reasonable, under some conditions, to consider from the start the associated integrable model of uncoupled pendulums subject to an effective external field. The authors of Ref. [43] found that the marginal distributions for this integrable model (IM) fit well the corresponding HMF qSS distributions for some initial conditions. We shall argue here that this will be the case only if the oscillations of the mean-field potential are negligible from the start, i.e., if the initial distribution satisfies the virial condition. The possibility of approximating the dynamics of the HMF by that of an IM further demonstrates that the hypothesis of ergodicity intrinsic to LB statistics is not valid.

In this paper we will compare the predictions of LB statistics and of the IM with extensive MD simulations of the HMF model with initial multilevel water-bag distributions satisfying the virial condition. In addition to the marginal distributions $\left[P_{\theta}(\theta)\right.$ and $\left.P_{p}(p)\right]$, we also calculate the energy distribution $f(\epsilon)$, which provides a sharper distinction among the different approaches [36,38]. With this comparison, we are interested in verifying whether the agreement observed between the LB theory and MD simulations for one-level distributions is fundamental or is simply a coincidence.

The paper is organized as follows: in Sec. II, we introduce the model and calculate the generalized virial condition for the multilevel ICs; in Sec. III we review the LB formalism; in Sec. IV, we present the IM of uncoupled pendula and use it to calculate the distribution functions for the qSS of the HMF; Sec. V is devoted to results and Sec. VI to conclusions.

\section{HMF MODEL}

The HMF is a paradigmatic model of a system with LRI [46,47]. The model was originally introduced to study the collective behavior observed in plasma and astrophysics. The model describes $N$ interacting particles constrained to move on a unit circle, or $N$ spins interacting through pairwise exchange interaction. The Hamiltonian of the ferromagnetic version of the model is given by (in units of the coupling constant)

$$
\mathcal{H}=\sum_{i} \frac{p_{i}^{2}}{2}+\frac{1}{2 N} \sum_{i, j=1}^{N}\left[1-\cos \left(\theta_{i}-\theta_{j}\right)\right],
$$

where $\theta_{i}$ is the position of the $i$ th particle on the unit circle and $p_{i}$ is its conjugate momentum. In equilibrium the model has a second-order phase transition between a homogeneous and an inhomogeneous bunched (ferromagnetic) state.

The one-particle energy is

$$
\epsilon(\theta, p)=\frac{p^{2}}{2}+1-M_{x} \cos (\theta)-M_{y} \sin (\theta),
$$


where $M=M_{x}+i M_{y}=1 / N \sum_{j=1}^{N} e^{i \theta_{j}}$ is the instantaneous magnetization, i.e., the average mean-field felt by any particle [see Eq. (2)], which in the case of the HMF is a position-independent function of time:

$$
M=\int d \theta d p f(\theta, p, t) e^{i \theta} .
$$

In this work we will explore the microcanonical dynamics of the HMF, which conserves the average energy per particle $u=\frac{\left\langle p^{2}\right\rangle}{2}+\frac{1-|M|^{2}}{2}$.

\section{A. Virial condition}

The determination of the virial condition for the HMF model poses a problem, since its interaction potential is not a homogeneous function of coordinates. This means that one cannot find a relation between the averages of kinetic and potential energy, and instead we are left with a functional equation which depends on the one-particle distribution function. To proceed we observe that in a stationary state the virial $G=\langle\mathbf{p} \cdot \mathbf{q}\rangle$ does not depend on time, so that

$$
\frac{d}{d t}\langle\mathbf{p} \cdot \mathbf{q}\rangle=\left\langle\frac{d}{d t}(\mathbf{p} \cdot \mathbf{q})\right\rangle=0 .
$$

Rewriting the above equation as

$$
\left\langle p^{2}\right\rangle=-\langle\mathbf{q} \cdot \ddot{\mathbf{q}}\rangle
$$

and considering a self-averaging system, i.e., that the above time averages are equivalent to averages over the particles, while making use of Hamilton's equations, it is easy to show the previous equation is equivalent to

$$
\left\langle p^{2}\right\rangle=-\frac{1}{N} \sum_{i=1}^{N} \mathbf{F}_{i} \cdot \mathbf{q}_{i} .
$$

Furthermore, in the case that interactions are long range and in the thermodynamic limit, the mean-field limit is exact, and thus the two-particle distribution function factorizes into a (one-particle) density distribution (pair correlations vanish) [2]. The virial theorem in this case reduces to

$$
\left\langle p^{2}\right\rangle=-\int d \mathbf{q}^{\prime} d \mathbf{p}^{\prime} f\left(\mathbf{q}^{\prime}, \mathbf{p}^{\prime}\right)\left[-\frac{\partial V\left(\mathbf{q}^{\prime}\right)}{\partial \mathbf{q}^{\prime}} \cdot \mathbf{q}^{\prime}\right],
$$

where $V\left(\mathbf{q}^{\prime}\right)$ is the mean-field potential, given by (2). The $\langle\cdot\rangle$ denotes the time average, which is equivalent to the ensemble average with a stationary measure. In the case of the HMF model, however, one is still left with a functional equation since the stationary distribution is not known. In Ref. [17], the authors propose a generalized virial condition for a water-bag initial distribution centered at $\theta=0\left(M_{y}=0\right)$, with the support $\left[-\theta_{m}, \theta_{m}\right] \times\left[-p_{1}, p_{1}\right]$ in phase-space. Using Eq. (10), $\left\langle p^{2}\right\rangle=-\frac{\sin \left(\theta_{m}\right) \cos \left(\theta_{m}\right)}{\theta_{m}}+\frac{\sin ^{2}\left(\theta_{m}\right)}{\theta_{m}^{2}}$. For the water-bag initial condition, $\langle p \theta\rangle(t=0)=0$, which implies that $\left\langle\theta^{2}\right\rangle$ remains constant for short times, so that the water-bag domain is not deformed and the relation $M=\frac{\sin \left(\theta_{m}\right)}{\theta_{m}}$ continues to hold. Replacing $\left\langle p^{2}\right\rangle$ by the corresponding function of $u$ and $M$, one ends up with the generalized virial condition for the HMF:

$$
(2 u-1) \theta_{m}+\cos \left(\theta_{m}\right) \sin \left(\theta_{m}\right)=0 .
$$

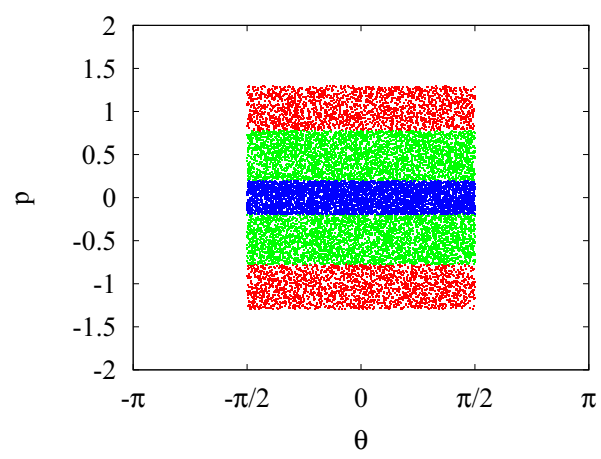

FIG. 1. (Color online) Phase-space representation of a multilevel initial condition. Different colors correspond to different phase space densities.

Equation (11) is only an approximation, since a water-bag distribution is not a stationary solution of the Vlasov equation. Nevertheless, the virial water-bag initial distribution should be "sufficiently close" to the final qSS to suppress any strong oscillations of the magnetization [17].

In this paper we will consider initial conditions uniform in $\theta$, and multilevel in $p$, given by

$$
\begin{aligned}
& f_{0}(\theta, p) \\
& \quad= \begin{cases}\eta_{1}, & \text { for } 0 \leqslant|p| \leqslant p_{1}, \\
\eta_{2}, & \text { for } p_{1}<|p| \leqslant p_{2}, \\
\vdots & \\
\eta_{L}, & \text { for } p_{L-1}<|p| \leqslant p_{L}, \\
0, & \text { for }|\theta| \leqslant \theta_{m},\end{cases}
\end{aligned}
$$

where $p_{1}<p_{2}<\cdots<p_{L-1}<p_{L}$ (see Fig. 1). For these ICs, the initial magnetization is always the same function of the envelope of the distribution, $\theta_{m}: M_{0}=\frac{\sin \left(\theta_{m}\right)}{\theta_{m}}$, so the virial condition will be the same as Eq. (11).

\section{LYNDEN-BELL STATISTICS}

Lynden-Bell suggested a statistical approach, based on a coarse-grained entropy [28], to try to account for the mass distribution in elliptical galaxies. Since the relaxation to qSS of elliptical galaxies is collisionless and is characterized by very strong oscillations of the mean-field potential, LB called this process violent relaxation.

A fundamental insight of LB's theory is that Vlasov flow is incompressible. This can be seen by recognizing that the volume in phase space occupied by a given phase space density level,

$$
v_{\eta}=\int d \mathbf{q} d \mathbf{p} \delta[f(\mathbf{q}, \mathbf{p}, t)-\eta],
$$

is a Casimir invariant [Eq. (3)] and is, therefore, preserved by the dynamics. Notice that this implies that the phase space density at later times cannot exceed the maximum of the density at time $t=0$.

Lynden-Bell argued that variations of the mean gravitational field to which particles (stars) are subjected are so violent that all phase space elements are equally likely to be found at a given energy. This is equivalent to requiring ergodicity and 
mixing. The relaxation of such systems should take place under the following constraints:

(1) That the total number of elements of phase with a given mass density (resp. magnetic moment, charge density, etc.) is conserved (incompressibility of Vlasov flow, no sinks, no sources).

(2) That total energy is conserved (isolated system).

(3) No overlap of two elements of phase is allowed by the dynamics, since in this case there would be no conservation of the number of phase elements as stated before (exclusion).

These three assumptions are a direct consequence of the Vlasov equation to which the dynamics of the timedependent phase space distribution function $f(\mathbf{q}, \mathbf{p}, t)$ is subjected. However, although very reasonable, the assumption of ergodicity does not have any a priori justification. This hypothesis, however, is crucial to LB statistics, since it allows a combinatorial counting of states, i.e., an "ensemble" approach to the distribution function.

LB defines a coarse-grained entropy, which is a functional of the coarse-grained one-particle density distribution $\bar{f}_{L B}(\mathbf{q}, \mathbf{p})=\sum_{\alpha=1}^{L} \bar{f}_{\alpha}(\mathbf{q}, \mathbf{p})$ and must be maximized with respect to $\left\{\bar{f}_{\alpha}\right\}$. The index $\alpha$ runs over the $L$ phase space levels, and $\bar{f}_{\alpha}(\mathbf{q}, \mathbf{p})$ are the respective level distributions. The most probable qSS should be the one which maximizes the entropy subject to the constraints of conservation of the phase space volumes of each level [Eq. (13)] and of the total energy

$$
\int\left(\frac{p^{2}}{2 m}+\frac{V(\mathbf{q})}{2}\right) \bar{f}_{L B}(\mathbf{q}, \mathbf{p}) d \mathbf{q} d \mathbf{p}=u .
$$

$V(\mathbf{q})$ is the average mean-field potential at point $q$ [Eq. (2) with $\left.f(\mathbf{q}, \mathbf{p}, t)=\bar{f}_{L B}(\mathbf{q}, \mathbf{p})\right]$. Since Vlasov dynamics does not lead to entropy production, entropy increase in the LB scheme is the result of coarse graining. Solving the variational problem, we obtain

$$
\bar{f}_{L B}(\mathbf{q}, \mathbf{p})=\sum_{\alpha=1}^{L} \eta_{\alpha} \frac{\exp \left[-\beta_{\alpha}\left(\epsilon(\{q\},\{p\})-\mu_{\alpha}\right)\right]}{1+\sum_{\gamma} \exp \left[-\beta_{\gamma}\left(\epsilon(\{q\},\{p\})-\mu_{\gamma}\right)\right]},
$$

where the $\eta_{\alpha}$ are the respective phase space level densities, and $\mu_{\alpha}$ and $\beta_{\alpha}=\beta \eta_{\alpha}$ are the effective chemical potentials and the inverse temperatures, which enforce the respective phase space volumes and energy conservation. The "inverse temperature" $\beta$ gives a measure of how degenerate the system is. In the limit of very low phase space density (high $\beta$ ), i.e., $f_{\alpha} \ll \eta_{\alpha}$, the LB distribution tends to a sum of Maxwellians.

The hypothesis of ergodicity "washes out" the memory of the initial condition, except for the conservation of the respective phase space volumes. Using the molecular dynamics simulations, in the forthcoming sections, we will show that conservation of the phase space volumes is not sufficient to predict the particle distribution in the qSS.

\section{INTEGRABLE MODEL ANALOGUE}

We now consider an approach that is diametrically opposite from the ergodicity-based LB statistics $[43,45]$. When the system attains a qSS the mean-field potential must be stationary, $V(\mathbf{q}, t)=V(\mathbf{q})$. Conversely, the mean-field potential can be stationary only if the density distribution function, $\rho(\mathbf{q}) \equiv \int d \mathbf{p} f(\mathbf{q}, \mathbf{p}, t)$, is also stationary [see Eq. (2)]. In this limit, particle movements uncouple, and $V(\mathbf{q})$ simply factors out as a constant (in the case of HMF, at least). Motivated by this, the authors in Refs. $[43,45]$ propose that the qSS of the HMF model might be well described by the qSS attained by an associated IM of uncoupled pendulums subject to a fixed external field $H$, whose single-particle energy function is given by

$$
\epsilon(\theta, p)=\frac{p^{2}}{2}+1-H \cos (\theta) .
$$

The value of the field $H$ is then fixed self-consistently to be $H=\langle\cos (\theta)\rangle=\frac{1}{N} \sum_{i} \cos \left(\theta_{i}\right)$. It was observed, however, that even though for some initial conditions this approximation leads to qSS distributions which are in a good agreement with the MD simulations, in general this is not the case. Indeed, from our previous discussion, we expect that the distribution function of uncoupled pendulums will provide a reasonable approximation to the qSS distribution of the HMF only if the oscillations of the mean-field potential are negligible from the start, i.e., when the initial particle distribution satisfies the virial condition. On the other hand, if the initial condition is far from virial, the parametric resonances will lead to the formation of a qSS with a characteristic core-halo structure $[13,17,48]$.

Determining the stationary distribution attained by the system of uncoupled pendulums is straightforward. Let's consider an arbitrary initial phase space distribution of angles and velocities. Evidently the dynamics of uncoupled pendulums is such that the number of pendulums with energy $[\varepsilon, \varepsilon+d \varepsilon]$ is a constant of motion. Since the force derived from Eq. (16) is nonlinear in angle, the particles on the energy shell $[\varepsilon, \varepsilon+d \varepsilon]$ with slightly distinct energies will have incommensurate orbital frequencies. Therefore, after a transient period, the resulting phase mixing will lead to a uniformity of the particle distribution over the energy shell. Suppose that we start with a distribution of angles and velocities $f_{0}(\theta, p)$. The number of particles with energy between $[\varepsilon, \varepsilon+d \varepsilon]$ is $n(\varepsilon) d \varepsilon$, where

$$
n(\varepsilon)=\iint d \theta d p f_{0}(\theta, p) \delta[\epsilon(\theta, p)-\varepsilon] .
$$

The density of states with a given energy $[\varepsilon, \varepsilon+d \varepsilon]$ is

$$
g(\varepsilon)=\iint d \theta d p \delta[\epsilon(\theta, p)-\varepsilon] .
$$

At $t=0$, however, not all of these states are occupied. Nevertheless, as the dynamics evolves, the phase mixing will result in a uniform occupation of all the states of a given energy, keeping $n(\varepsilon)$ constant. The coarse-grained distribution function for the stationary state of a system of uncoupled pendulums $\bar{f}(\varepsilon)$ must then satisfy $\bar{f}(\varepsilon) g(\varepsilon)=n(\varepsilon)$, from which we conclude that

$$
\bar{f}(\varepsilon)=\frac{\iint d \theta d p f_{0}(\theta, p) \delta[\epsilon(\theta, p)-\varepsilon]}{\iint d \theta d p \delta[\epsilon(\theta, p)-\varepsilon]} .
$$

The density of states can be calculated explicitly to be [36]

$$
g(\varepsilon)= \begin{cases}4 K\left(\kappa^{1 / 2}\right) / \sqrt{H}, & \text { if } \kappa \leqslant 1, \\ 4 K\left(\kappa^{-1 / 2}\right) / \sqrt{H \kappa}, & \text { if } \kappa>1,\end{cases}
$$


where $\kappa(\varepsilon ; H)=(\varepsilon-1+H) / 2 H$, and $K(x)$ is the complete elliptic integral of the first kind (defined as in Ref. [49]). Again it is important to stress that the formalism above applies only to the IM of uncoupled pendulums. In particular we see that the initial occupation of the energy shells is preserved throughout the dynamics. The initially unpopulated energy shells will remain unpopulated in the stationary distribution derived above. This, in general, is not the case for the HMF model away from the virial condition when the parametric resonances lead to the occupation of the high-energy states not present in the initial distribution. On the other hand, if the virial condition is satisfied, $M(t)$ will remain constant (in the thermodynamic limit) and the parametric resonances will be suppressed. In this case IM with $H=M(0)=M_{0}$ should provide an accurate description of the stationary distribution.

To calculate the coarse-grained distribution for the stationary state starting from an initial water-bag distribution $f_{0}(\theta, p)=\frac{1}{4 \theta_{m} p_{1}} \Theta\left(\theta_{m}-|\theta|\right) \Theta\left(p_{1}-|p|\right)$, we must perform the integration over the phase space in the numerator of Eq. (19). Integrating first over momentum, we find

$$
\begin{aligned}
2 \eta_{1} \int d \theta \Theta\left(\theta_{m}-|\theta|\right) \int_{0}^{p_{1}} d p \delta\left\{\frac{p^{2}}{2}-[\varepsilon-1+H \cos (\theta)]\right\} \\
=2 \sqrt{2} \eta_{1} \int_{0}^{\theta_{m}} d \theta \frac{\Theta[\varepsilon-1+H \cos (\theta)]}{\sqrt{\varepsilon-1+H \cos (\theta)}} \\
\quad \times \Theta\left\{\frac{p_{1}^{2}}{2}-[\varepsilon-1+H \cos (\theta)]\right\},
\end{aligned}
$$

where $\eta_{1}=\frac{1}{4 \theta_{m} p_{1}}$ is the normalization constant of the density distribution function $f_{0}$. The theta functions in the integrand above impose restrictions on the domain of integration over $\theta$. Performing the integration in $\theta$, the numerator can then be written as

$$
\begin{aligned}
& \iint d \theta d p f_{0}(\theta, p) \delta[\epsilon(\theta, p)-\varepsilon] \\
& =\frac{1}{\theta_{m} p_{1}} \frac{1}{\sqrt{H \kappa}}\left[F\left(\frac{\theta_{\mathrm{up}}^{(1)}}{2}, \kappa^{-1 / 2}\right)-F\left(\frac{\theta_{\mathrm{low}}^{(1)}}{2}, \kappa^{-1 / 2}\right)\right],
\end{aligned}
$$

where $F(\phi, x)$ is the incomplete elliptic integral of the first kind, and the appropriate limits of integration $\theta_{\mathrm{low}}^{(1)}$ and $\theta_{\mathrm{up}}^{(1)}$ are defined below.

The $L$-level initial conditions defined by Eq. (12) may be considered as a superposition of water bags with different supports. Superposition of result (19) is possible since the pendulums are noninteracting. For these ICs the contribution to the numerator of Eq. (19) from each of the $L$ water bags of the multilevel IC gives

$$
I^{(i)}(\varepsilon ; H) \equiv \frac{4 \sqrt{2}}{\sqrt{H \kappa}}\left[F\left(\frac{\theta_{\mathrm{up}}^{(i)}}{2}, \kappa^{-1 / 2}\right)-F\left(\frac{\theta_{\mathrm{low}}^{(i)}}{2}, \kappa^{-1 / 2}\right)\right],
$$

where the superscript $(i)$ refers to a water bag with domain $\left[-\theta_{m}, \theta_{m}\right] \times\left[-p_{i}, p_{i}\right]$. The upper and lower limits of integra-

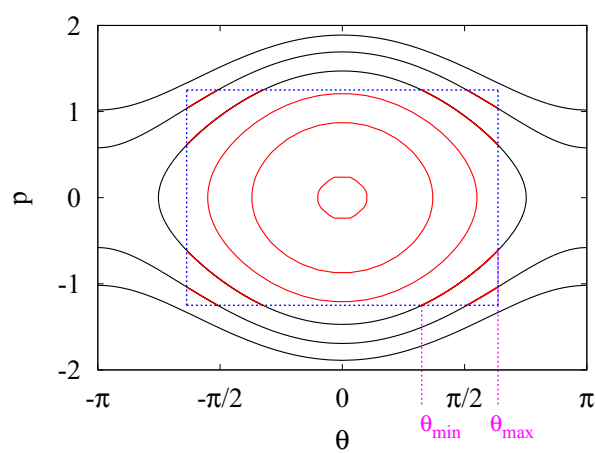

FIG. 2. (Color online) Schematic representation of the construction of the final distribution function for the uncoupled pendulums. Superimposed is the support of the water-bag initial condition. In red are the orbits (chunks of orbits) that contribute to the integral (19).

tion are determined by (see Fig. 2)

$\theta_{\text {up }}^{(i)}=\min \left\{\cos ^{-1}(1-2 \kappa), \theta_{m}\right\}$

$\theta_{\text {low }}^{(i)}= \begin{cases}0, & \text { for } \epsilon \leqslant p_{i}^{2} / 2-H, \\ \min \left\{\cos ^{-1}\left(\frac{p_{i}^{2}}{2 H}+1-2 \kappa\right), \theta_{m}\right\}, & \text { for } \epsilon>p_{i}^{2} / 2-H .\end{cases}$

Of course, $I^{(i)}(\varepsilon ; H)=0$ for energies $\varepsilon \leqslant 1-H$ or $\varepsilon \geqslant$ $\varepsilon_{\max }=p_{i}^{2} / 2+1-H \cos \left(\theta_{m}\right)$ [from Eq. (24)]. The singleparticle distribution function is then given by

$$
\bar{f}(\varepsilon)=\frac{\eta_{L} I^{(L)}+\sum_{i=1}^{L-1}\left(\eta_{L-i}-\eta_{L+1-i}\right) I^{(L-i)}}{g(\varepsilon)} .
$$

\section{NUMERICAL RESULTS}

To explore the validity of the theory constructed above we have performed molecular dynamics simulations of the HMF model with $N=10^{6}$ particles. The system is allowed to relax until a qSS is reached. We then compute the position and momentum marginal distribution functions $P_{\theta}(\theta)$ and $P_{p}(p)$, as well as the energy distribution $\bar{f}(\varepsilon)$ [36]. In Figs. 3-5 and $8-10$ we compare the predictions of LB theory and of IM with the results of MD simulations for water-bag initial distributions with increasing number of levels $L$.
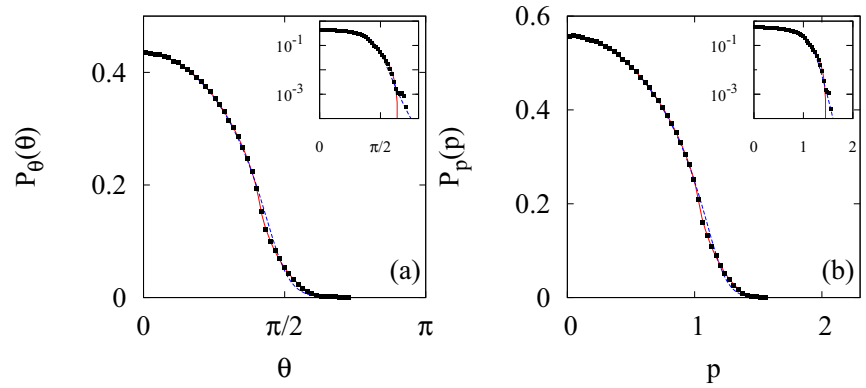

FIG. 3. (Color online) Comparison of the marginal distributions in (a) angles and (b) velocities with the results of MD simulations for the qSS of the HMF. The LB distribution (blue dashed curves) and the IM distribution (red solid curves) for a virial one-level water-bag initial condition with $u=0.4, M_{0}=0.742$. 

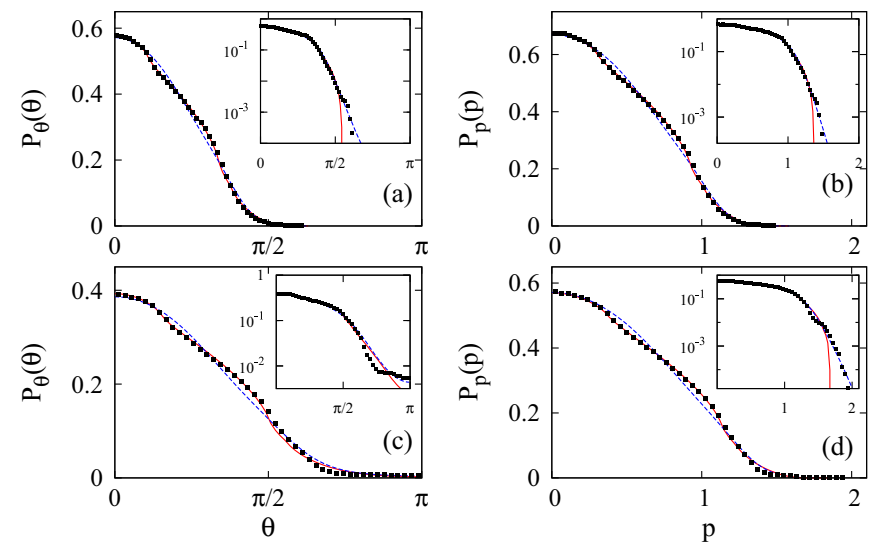

FIG. 4. (Color online) Comparison of the marginal qSS distributions in angle [(a) and (c)] and momentum [(b) and (d)] of the MD (black squares), the corresponding LB stationary distribution (blue dashed curves), and IM distribution (red solid curves), for two sets of virial two-level water-bag initial conditions. (a) and (b) $u=0.3$, initial magnetization $M_{0}=0.822, \theta_{m}=1.06, p_{1}=1.02$, $\eta_{1}=0.106$; (c) and (d) $u=0.5, M_{0}=0.637, \theta_{m}=1.58, p_{1}=1.24$, $\eta_{1}=0.059$. For both ICs $p_{1}=0.3 p_{2}, \eta_{2}=0.5 \eta_{1}$.

\section{A. Marginal distributions}

Figures 3-5 show the marginal distributions of angles (left panels) and velocities (right panels), which are calculated from the full single-particle density distribution function $f(\theta, p)$ as

$$
P_{\theta}(\theta) \equiv \int d p f(\theta, p) ; \quad P_{p}(p) \equiv \int d \theta f(\theta, p) .
$$

Figure 3 is for a one-level water-bag initial condition; Fig. 4, for a two-level IC; and Fig. 5, for a three-level IC. We note the departure of both IM and LB from MD for increasing number of density levels $L$.
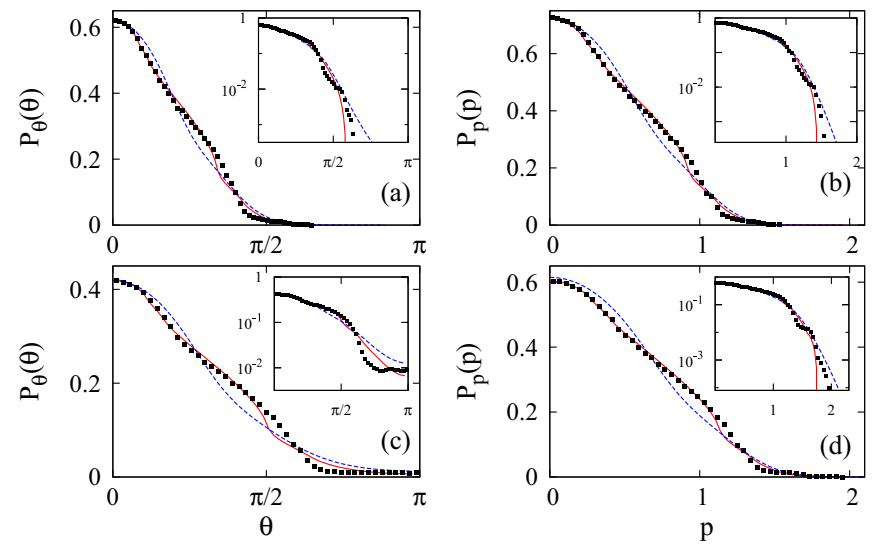

FIG. 5. (Color online) Comparison of the marginal qSS distributions in angle [(a) and (c)] and momentum [(b) and (d)] of the MD (black squares), the corresponding LB stationary distribution (blue dashed curves), and IM distribution (red solid curves), for two sets of virial three-level water-bag initial conditions. (a) and (b) $u=0.3$, initial magnetization $M_{0}=0.822, \theta_{m}=1.06, p_{1}=1.12$, $\eta_{1}=0.079 ;$ (c) and (d) $u=0.5, M_{0}=0.637, \theta_{m}=1.58, p_{1}=1.35$, $\eta_{1}=0.044$. For both ICs $p_{1}=0.3 p_{2}, \eta_{2}=0.5 \eta_{1}, p_{3}=0.2 p_{1}, \eta_{3}=$ $0.3 \eta_{1}$.
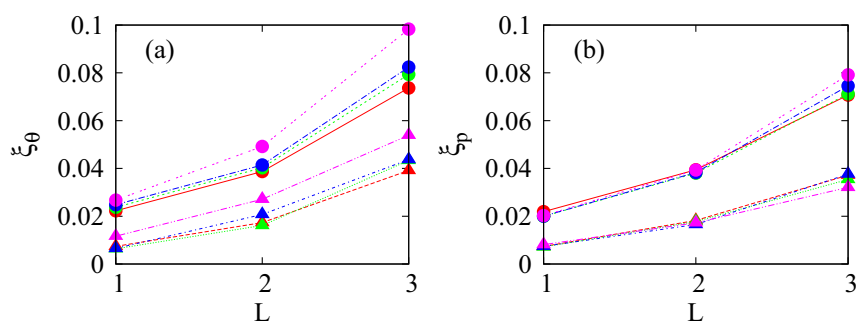

FIG. 6. (Color online) The rms deviations $\xi_{\theta}$ and $\xi_{p}$ between the LB (IM) marginal distributions in angle and momentum, respectively, and the MD distributions, as a function of number of levels $L$. Triangles correspond to IM-MD deviation, $\xi^{I M}$, and circles to IM-MD deviation, $\xi^{L B}$. Red symbols (triangles and circles) correspond to $u=0.2$; green symbols, to $u=0.3$; blue symbols, to $u=0.4$; and pink symbols, to $u=0.5$.

Nevertheless, IM accounts very accurately for the MD data; see Figs. 3-5. The differences are noticeable only in the tails of the distributions. Although for one-level initial conditions LB theory provides an accurate description of the marginal distributions, this agreement deteriorates rapidly for multilevel initial conditions. The discrepancy between LB and MD simulations is clearest when one considers the complete distribution function $\bar{f}(\varepsilon)$. In the next section we will see
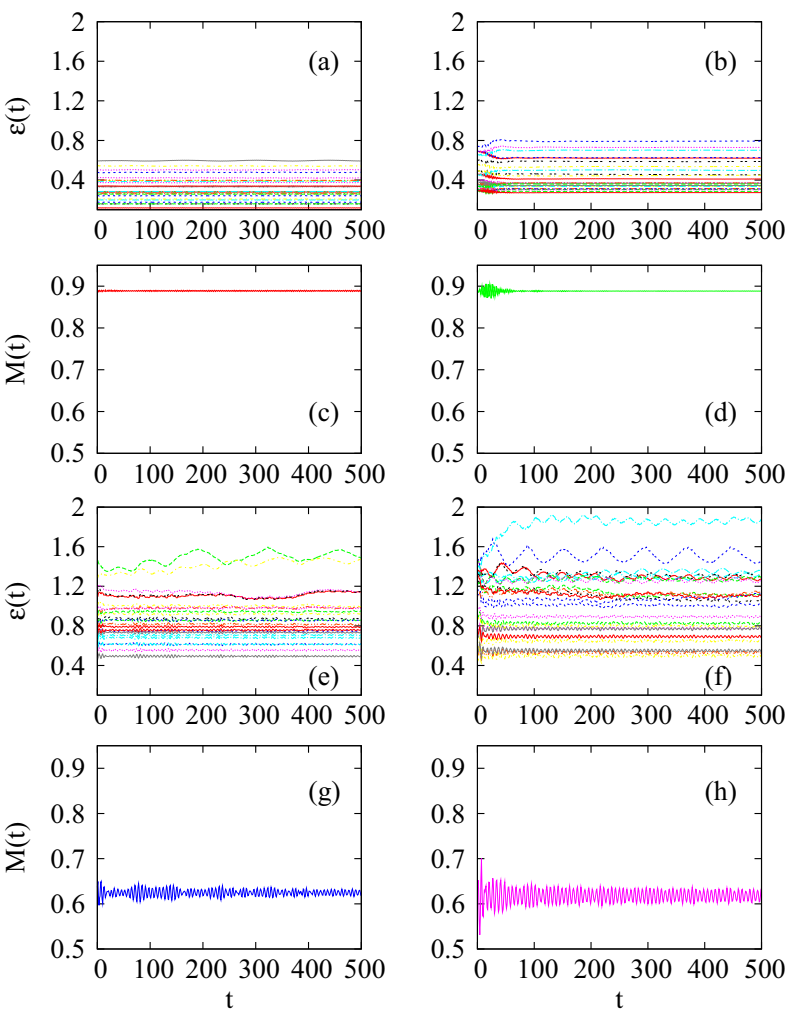

FIG. 7. (Color online) Comparison between the one-particle energies for a few selected particles and corresponding magnetization as a function of time. Panels (a) and (c) correspond to $u=0.2$, $L=1$; panels (b) and (d), to $u=0.2, L=3$; panels (e) and (g), to $u=0.5, L=1$; panels (f) and (h), to $u=0.5, L=3$. Note the presence of significant oscillations both in one-particle energy and in magnetization, for multilevel distributions. 

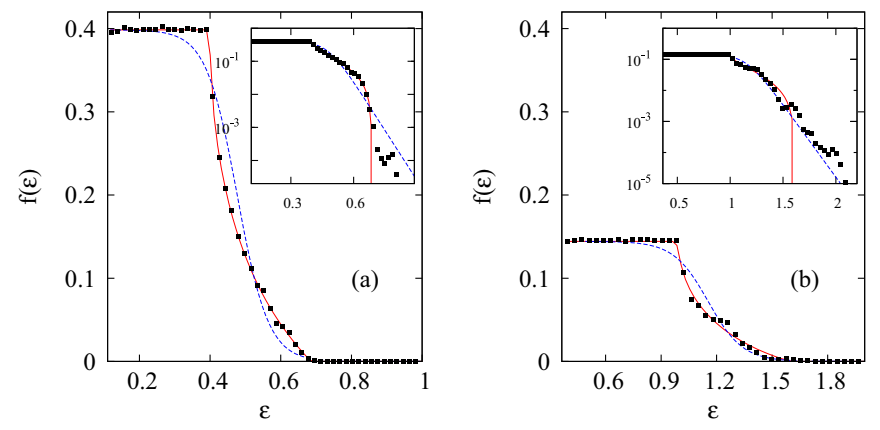

FIG. 8. (Color online) Comparison between MD qSS energy distribution function (black squares), the corresponding LB distribution (blue dashed curve), and IM stationary distribution (red solid curve), for a one-level water-bag IC; (a) $u=0.2$; (b) $u=0.5$. Insets are the same curves in log-linear scale. The same IC parameters as in Fig. 3.

that, although the simulations show the existence of a fully degenerate core, LB theory predicts a nondegenerate core characterized by a finite Fermi-Dirac temperature.

To quantify the extent of agreement between the MD simulations and IM and LB theories we define the root-meansquare (rms) deviation of $f_{2}(x)$ from a reference distribution $f_{1}(x)$ as

$$
\xi_{x}=\left\{\frac{\int d x\left[f_{2}(x)-f_{1}(x)\right]^{2}}{\int d x f_{1}^{2}(x)}\right\}^{1 / 2} .
$$

In Fig. 6 we plot $\xi_{x}$ for IM-MD (triangles) and for LB-MD (circles) for marginal distributions in angle and momentum. Although $\xi$ do not exceed $10 \%$, we see that for fixed $L$, the deviations between LB-MD are always greater than deviations between IM-MD. For given $L$ and $u$, rms deviation between LB-MD is roughly twice the corresponding deviation between IM-MD. Therefore, the HMF qSS is closer to a completely integrable system than to the ergodicity based LB theory. Furthermore, since both $\xi^{I M}$ and $\xi^{L B}$ (either for $P_{\theta}$ or $P_{p}$ ) increase with increasing number of levels, results suggest that for general ICs, HMF qSS will be neither ergodic nor integrable.
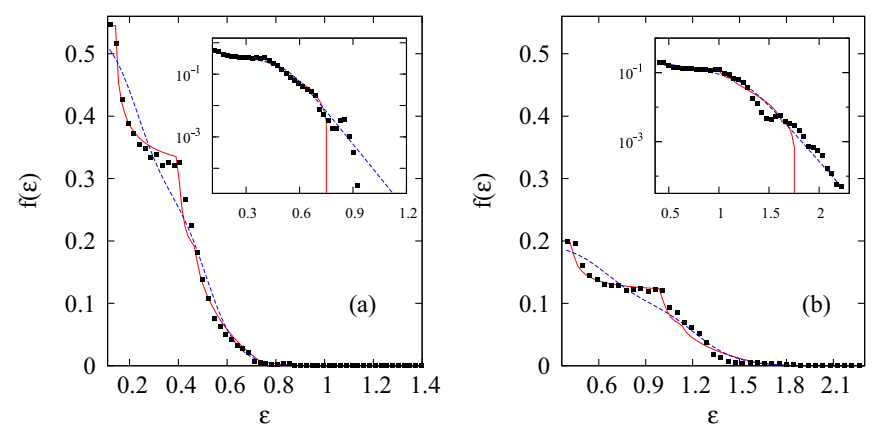

FIG. 9. (Color online) Comparison between MD qSS energy distribution function (black squares), the corresponding LB distribution (blue dashed curve), and IM stationary distribution (red solid curve) for a two-level IC; (a) $u=0.2$; (b) $u=0.5$. The same IC parameters as in Fig. 4.
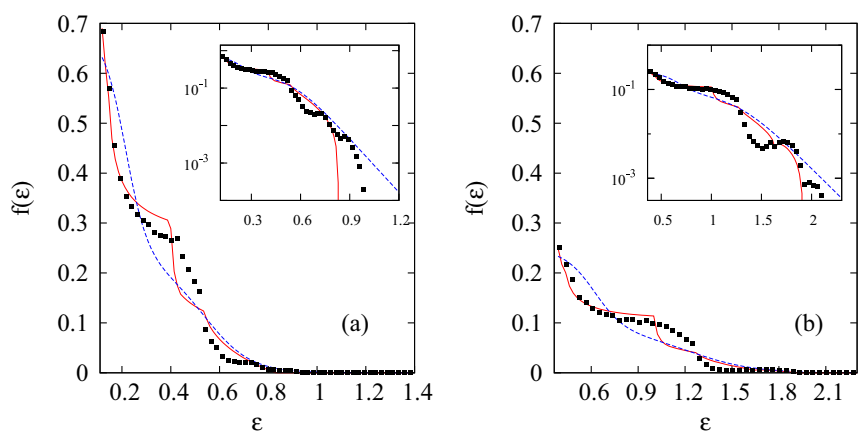

FIG. 10. (Color online) Comparison between MD qSS energy distribution function (black squares), the corresponding LB distribution (blue dashed curve), and IM analog stationary distribution (red solid curve) for a three-level IC; (a) $u=0.2$; (b) $u=0.5$. The same IC parameters as in Fig. 5.

The departure from IM predictions, in particular, is a consequence of the fact that, when increasing $L$, the virial condition (11) does not restrict the stationarity of all the density levels. This is clearly shown in Fig. 7, where we plot, side-by-side, single-particle energies' time evolution (for some selected particles of the system) and the time evolution of the system magnetization. There is a clear relationship between the amplitude of the oscillations of the magnetization and both the variation and variance of the single-particle orbit energies. It demonstrates that for multilevel water-bag distributions satisfying the generalized virial condition, both one-particle energies and magnetization undergo significant oscillations. These oscillations are expected to give rise to parametric resonances which will lead to the halo formation.

\section{B. Energy distributions}

The integration over angles (momentums) required to calculate the marginal distributions $P_{p}\left(P_{\theta}\right)$ smoothes out these functions. To explore better the one-particle distribution in the $\mathrm{qSS}$ it is, therefore, important to study the full distribution function $\bar{f}(\varepsilon)$ :

$$
\bar{f}(\varepsilon)=\frac{\int d \theta d p f(\theta, p) \delta[\epsilon(\theta, p)-\varepsilon]}{\int d \theta d p \delta[\epsilon(\theta, p)-\varepsilon]},
$$

where $\epsilon(\theta, p)$ is the single-particle energy function (5) and the denominator is the density of states $g(\varepsilon)$.

Figure 8 shows the comparison between MD, IM, and LB energy distributions for a one-level water-bag IC. Figure 9 shows the same for a two-level IC, and Fig. 10 for a three-level IC. Here again the discrepancy between IM predictions and the MD data is larger in the tails of the distributions. Even though the system starts on the virial curve (11), the water-bag IC is not a fixed point of the Vlasov dynamics. Therefore, for multilevel distributions the magnetization (mean-field potential) may undergo significant oscillations. In this case, some particles may enter in resonance with the mean-field oscillations gaining energy to form a tenuous halo.

For one-level water-bag initial conditions, we see that the MD-obtained energy distribution is in very good agreement 

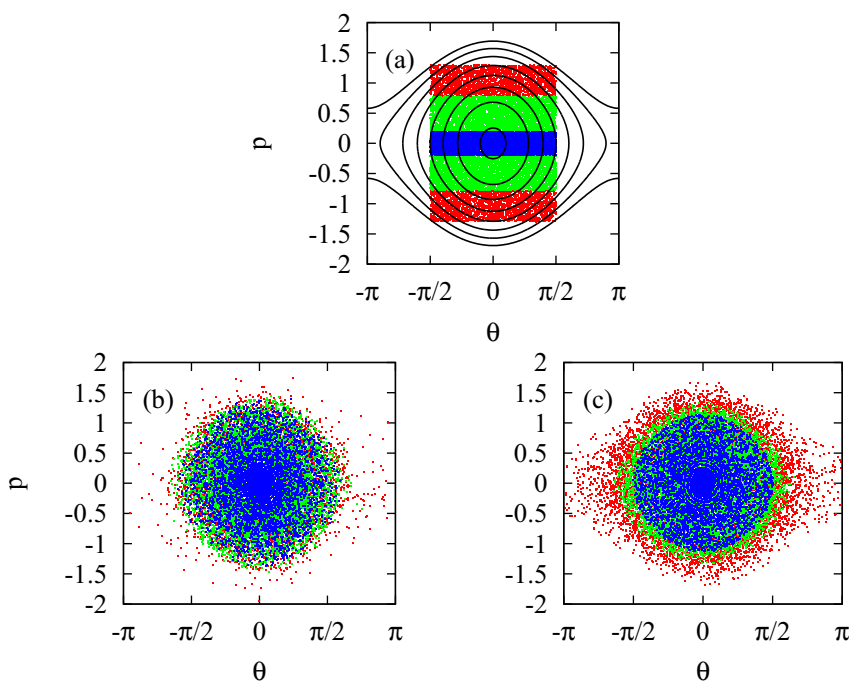

FIG. 11. (Color online) Snapshots of the phase space at (a) $t=0$ (three-level IC); (b) $t=1.7 \times 10^{4}$ time steps after evolution under Eq. (4) (HMF model); (c) $t=10^{3}$ time steps after evolution under Eq. (16) (uncoupled pendulums). In both cases (b) and (c) systems have attained stationarity. The number of points is the same in all panels.

with the predictions of IM. On the other hand LB theory is incompatible with MD data (see Fig. 8), while MD shows a plateau at the maximum phase-space density, corresponding to a fully degenerate Fermi core [48], LB predicts a smoothly decaying function, corresponding to a Fermi-Dirac distribution with a finite temperature.

For a two-level IC (Fig. 9), we see that the distribution function predicted by the IM deviates from the results of MD simulations. The qualitative structure, however, is maintained. In particular, a small (for the specific set of chosen parameters) plateau persists for low energies, as well as a two-step decay of $\bar{f}(\varepsilon)$, which reflects the two-level IC. While MD shows that the occupation of the lowest energy levels is the same as in the initial distribution, LB predicts a lower maximum density for the qSS. The perfect mixing required by LB allows for initially more energetic levels to decay and penetrate the region originally occupied by the less energetic levels, contrary to what is seen in numerical simulations [36].

For the three-level IC (Fig. 10), we see that the energy distribution starts to deviate significantly from predictions of IM. This is the result of mixing between the different density levels, which within IM is possible only among the initial levels lying on the same trajectory (orbit) (see Fig. 11). Nevertheless, we see that for lower energies, IM and MD curves still coincide. One might think that increased mixing between the different phase space density levels will lead to an improved agreement between LB theory and MD simulations. This, however, is not the case, and, in particular, we see that for three-level distributions LB theory provides an even poorer fit of MD data than for two levels.

One may notice that for higher average energies $u$ [insets in the (b) panels in Figs. 8-10] the tails of the distributions, which lie close to the separatrix energy $\varepsilon_{s x}=1+M_{0}$, are closer to LB distribution. For these values of $u$, the separatrix is occupied by the initial distribution $f_{0}(\theta, p)$. Since at the sep-

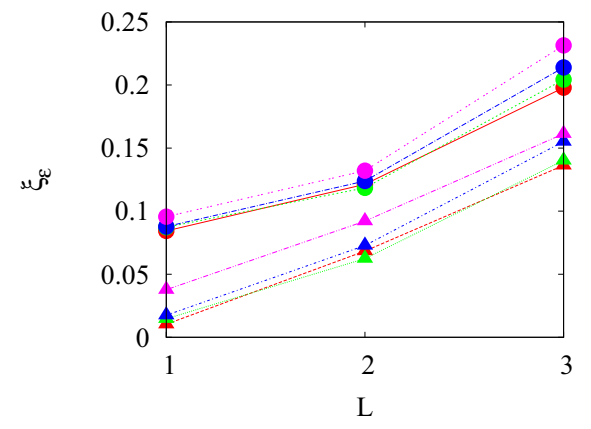

FIG. 12. (Color online) The rms deviations $\xi_{\varepsilon}$ between LB (IM) energy distributions and the corresponding MD distribution, as a function of number of levels $L$. Triangles correspond to IM-MD rms deviation, $\xi_{\varepsilon}^{I M}$, and circles to IM-MD rms deviation, $\xi_{\varepsilon}^{L B}$. Red symbols (triangles and circles) correspond to $u=0.2$; green symbols, to $u=0.3$; blue symbols, to $u=0.4$; and pink symbols, to $u=0.5$.

aratrix the resonance criterion is met by mean-field oscillations of any frequency (and infinitesimal amplitude) [50], particles initially at $\left[\varepsilon_{s x}-\delta \varepsilon, \varepsilon_{s x}+\delta \varepsilon\right]$ will be excited, and the tails of $\bar{f}(\varepsilon)$ will not coincide with the distribution given by the IM approximation. Even though the LB distribution allows for particle excitation, we argue that similarity with the tails of the numerical data is incidental, since the particles forming the tails of the MD distributions represent a fraction of $\lesssim 0.1 \%$ of the total system. Moreover, we argue that the similar behavior observed at the tails of the marginal distributions (Figs. 3-5) should be reminiscent of the same phenomenon, since the marginals $P_{\theta}(\theta)$ and $P_{p}(p)$ are derived from the full energy distribution $\bar{f}(\varepsilon)$ through integration over $p$ or $\theta$, respectively.

In Fig. 12 we compare the rms deviation $\xi_{\varepsilon}^{I M}$ (triangles) and $\xi_{\varepsilon}^{L B}$ (circles) as a function of the number of levels in the IC, for different values of the average energy per particle $u$. Once again we see that for a given $L$, the rms deviation $\xi_{\varepsilon}^{I M}$ is always below $\xi_{\varepsilon}^{L B}$. Furthermore, sensitivity to $u$ is much milder than sensitivity to $L$. Specially for the more complex ICs (two and three levels), the relative departure of LB and IM predictions for the energy distributions is significantly larger than it is for the marginal distributions, being close to $25 \%$ for $L=3\left(\xi_{\varepsilon}^{L B}\right)$.

\section{CONCLUSIONS}

We have computed, using extensive MD simulations, the qSS distribution functions of the HMF model starting from the multilevel water-bag initial conditions. The distributions were compared with the predictions of the Lynden-Bell theory and a theory based on the coarse-grained dynamics of uncoupled pendulums, the integrable model (IM) $[28,44]$. To suppress the halo formation all the initial water-bags were chosen to satisfy the generalized virial condition [17].

It is important to stress that LB and IM theories are based on diametrically opposite assumptions. While LB requires ergodicity and good mixing, IM is completely nonergodic. We have verified that for all sets of parameters considered, the HMF model was closer to the integrable limit than to the LB-postulated ergodicity. We have also verified that as 
the initial conditions become more complex, i.e., as the number of levels increases, the distribution functions deviate stronger from the predictions of both LB and IM theories. Nevertheless for all the cases investigated, IM remained more accurate than LB theory. The challenge for the future is to understand the structure of the qSS for the multilevel distribution functions, which do not satisfy the virial condition. For one-level water bags the core-halo theory provides a very accurate description of the structure of such qSS [13]. For multilevel systems, however, the complicated mixing between the different phase-space density levels results in a very complex evolution of the core, which so far has escaped any simple characterization.

\section{ACKNOWLEDGMENTS}

The authors acknowledge discussions with Bruno Marcos and Tarcísio N. Teles. We also acknowledge support from CFCIF (IF/UFRGS) for computational resources. This work was partially supported by FAPERGS, CAPES, CNPq, and INCT-FCx and by the US-AFOSR under Grant No. FA955012-1-0438.
[1] T. Padmanabhan, Phys. Rep. 188, 285 (1990).

[2] T. N. Teles, Y. Levin, R. Pakter, and F. B. Rizzato, J. Stat. Mech. Theor. Exp. (2010) P05007.

[3] T. Teles, Y. Levin, and R. Pakter, Mon. Not. R. Astron. Soc. 417, L21 (2011).

[4] B. B. Kadomtsev and O. P. Pogutse, Phys. Rev. Lett. 25, 1155 (1970).

[5] Y. Levin, R. Pakter, and T. N. Teles, Phys. Rev. Lett. 100, 040604 (2008).

[6] J. Barré, T. Dauxois, G. De Ninno, D. Fanelli, and S. Ruffo, Phys. Rev. E 69, 045501 (2004).

[7] A. Antoniazzi, Y. Elskens, D. Fanelli, and S. Ruffo, Eur. Phys. J. B 50, 603 (2006).

[8] F. Bouchet and A. Venaille, Phys. Rep. 515, 227 (2012).

[9] M. Chalony, J. Barré, B. Marcos, A. Olivetti, and D. Wilkowski, Phys. Rev. A 87, 013401 (2013).

[10] E. Shahmoon, I. Mazets, and G. Kurizki, arXiv:1309.0555.

[11] T. Dauxois, S. Ruffo, and L. F. Cugliandolo, editors, Long-Range Interacting Systems, Lecture Notes of the Les Houches Summer School, Vol. 90 (Oxford University Press, Oxford, 2008).

[12] A. Campa, T. Dauxois, and S. Ruffo, Phys. Rep. 480, 57 (2009).

[13] Y. Levin, R. Pakter, F. B. Rizzato, T. N. Teles, and Fernanda P. da C. Benetti, Phys. Rep. 535, 1 (2014).

[14] T. Tsuchiya, T. Konishi, and N. Gouda, Phys. Rev. E 50, 2607 (1994).

[15] F. Borgonovi, G. L. Celardo, M. Maianti, and E. Pedersoli, J. Stat. Phys. 116, 1435 (2004).

[16] D. Mukamel, S. Ruffo, and N. Schreiber, Phys. Rev. Lett. 95, 240604 (2005).

[17] Fernanda P. da C. Benetti, T. N. Teles, R. Pakter, and Y. Levin, Phys. Rev. Lett. 108, 140601 (2012).

[18] V. Latora, A. Rapisarda, and S. Ruffo, Phys. Rev. Lett. 83, 2104 (1999).

[19] V. Latora, A. Rapisarda, and S. Ruffo, Physica A 280, 81 (2000).

[20] V. Latora, A. Rapisarda, and S. Ruffo, Phys. Rev. Lett. 80, 692 (1998).

[21] Y. Yamaguchi, J. Barré, F. Bouchet, T. Dauxois, and S. Ruffo, Physica A 337, 36 (2004).

[22] P. Hertel and W. Thirring, Ann. Phys. 63, 520 (1971).

[23] J. Barré, D. Mukamel, and S. Ruffo, Phys. Rev. Lett. 87, 030601 (2001).
[24] O. Cohen and D. Mukamel, J. Stat. Mech. Theor. Exp. (2012) P12017.

[25] W. Thirring, Z. Phys. 235, 339 (1970).

[26] D. Lynden-Bell, Physica A 263, 293 (1999).

[27] W. Braun and K. Hepp, Commun. Math. Phys. 56, 101 (1977).

[28] D. Lynden-Bell, Mon. Not. R. Astr. Soc. 136, 101 (1967).

[29] P. Chavanis and F. Bouchet, Astron. Astrophys. 430, 771 (2005).

[30] T. M. Rocha Filho, A. Figueiredo, and M. A. Amato, Phys. Rev. Lett. 95, 190601 (2005).

[31] Y. Y. Yamaguchi, Phys. Rev. E 78, 041114 (2008).

[32] S. Goldstein, S. Cuperman, and M. Lecar, Mon. Not. R. Astr. Soc. 143, 209 (1969).

[33] S. Cuperman, S. Goldstein, and M. Lecar, Mon. Not. R. Astr. Soc. 146, 161 (1969).

[34] M. Lecar and L. Cohen, Astrophys. Space Sci. 13, 397 (1971).

[35] M. Joyce and T. Worrakitpoonpon, Phys. Rev. E 84, 011139 (2011).

[36] R. Pakter and Y. Levin, Phys. Rev. Lett. 110, 140601 (2013).

[37] R. Pakter and Y. Levin, J. Stat. Phys. 150, 531 (2013).

[38] A. Campa and P.-H. Chavanis, Eur. Phys. J. B 86, 170 (2013).

[39] Y. Levin, R. Pakter, and F. B. Rizzato, Phys. Rev. E 78, 021130 (2008).

[40] C.-S. Wu, Phys. Rev. 127, 1419 (1962).

[41] D. Sagan, Am. J. Phys. 62, 450 (1994).

[42] L. D. Landau, J. Phys. (USSR) 10, 25 (1946).

[43] P. de Buyl, D. Mukamel, and S. Ruffo, Philos. Trans. R. Soc. London, Ser. A 369, 439 (2011).

[44] P. de Buyl, D. Mukamel, and S. Ruffo, Phys. Rev. E 84, 061151 (2011).

[45] X. Leoncini, T. L. Van Den Berg, and D. Fanelli, Europhys. Lett. 86, 20002 (2009).

[46] T. Konishi and K. Kaneko, J. Phys. A 25, 6283 (1992).

[47] M. Antoni and S. Ruffo, Phys. Rev. E 52, 2361 (1995).

[48] R. Pakter and Y. Levin, Phys. Rev. Lett. 106, 200603 (2011).

[49] I. S. Gradshteyn and I. M. Ryzhik, Table of Integrals, Series and, Products (Academic Press, New York, 2007).

[50] A. Lichtenberg and A. Lieberman, Regular and Chaotic Dynamics, Applied Mathematical Sciences (Springer, New York, 2010). 